\title{
Irradiation system for pre-clinical studies with laser accelerated electrons
}

\author{
M. Schürer ${ }^{1}$, M. Baumann ${ }^{1,2}$, E. Beyreuther ${ }^{3}$, K. Brüchner ${ }^{1}$, W. Enghardt ${ }^{1,2,3}$, M. Kaluza ${ }^{4}$, L. Karsch ${ }^{1}$, L. Laschinsky ${ }^{1}$, \\ E. Leßmann ${ }^{3}$, M. Nicolai ${ }^{4}$, M. Oppelt ${ }^{1}$, M. Reuter ${ }^{4}$, C. Richter ${ }^{1,3}$, A. Sävert ${ }^{4}$, M. Schnell ${ }^{4}$, J. Woithe ${ }^{1}$ and J. Pawelke ${ }^{1,3}$ \\ ${ }^{1}$ OncoRay - National Center for Radiation Research in Oncology, Technische Universität, Dresden, Germany \\ ${ }^{2}$ Clinic of Radiotherapy and Radiooncology, Universitätsklinikum Carl Gustav Carus, Dresden, Germany \\ ${ }^{3}$ Institute of Radiation Physics, Helmholtz-Zentrum Dresden-Rossendorf (HZDR), Dresden, Germany \\ ${ }^{4}$ Institute of Optics and Quantum Electronics, Friedrich-Schiller-Universität (FSU), Jena, Germany
}

\begin{abstract}
In recent years, the new technology of laser based particle acceleration was developed at such a rate that medical application for cancer therapy could become feasible. Promising more compact and economic proton and ion accelerators the laser technology however results in specific properties, like ultra-short ( $\sim$ ps) and ultra-intensive particle beam pulses. The clinical applicability of such new beam qualities requires comprehensive translational research from basic investigations to cell and animal experiments, finally followed by clinical trials. For the first time, the new laser based irradiation technology was established for animal experiments by the German joint research project “onCOOPtics”. A complete irradiation facility for laser accelerated electrons was developed, set up, commissioned, tested and applied for radiobiological tumour irradiation experiments under usage of a mouse model at the high intensity laser system JETI. The integration of a magnet and a collimator system resulted in an optimized beam transport and efficient electron energy filtration. Moreover, a specific irradiation and dosimetry setup was integrated allowing for the formation of irradiation fields, the real-time control of beam parameters and dose delivery to the tumour. For an accurate and reproducible positioning of the tumour in the irradiation field the mice were fixed in a movable box and the tumour position was online verified by means of a CCD camera system. The combination of both, the advanced laser accelerator system and the newly implemented irradiation and dosimetry setup allowed the successful performance of systematic radiobiological studies over months. Moreover, the practicability and easy handling of the system results in a reasonable duration of about 15 min for the whole procedure of mouse preparation, positioning and irradiation. In conclusion, the successful establishment of all technical requirements for and the performance of systematic animal studies with laser accelerated electrons mark an important step towards the clinical application of laser accelerated particle beams.
\end{abstract}

\section{Introduction}

In recent years, the technological progress achieved for the new approach of laser based particle acceleration was at such a rate, that medical application, i.e. for cancer therapy, becomes conceivable. The usage of high intensity lasers promises more compact and less expensive proton and ion accelerators which could be integrated in existing hospitals to enable a more widespread application of this kind of high precision radiation therapy.

However, in contrast to conventionally accelerated, quasi continuous particle beams the laser accelerated particle beams are characterized by ultra-short beam pulses in the femtosecond to picosecond regime that are delivered with ultra-high dose per pulse at low repetition rate of typically a few Hz. Further specific properties of laser accelerated particle beams are the rather large beam divergence and the broad distribution of beam energy. Therefore, beam transport, irradiation field formation, beam monitoring and dosimetry have to be developed for laser accelerated particle pulses. Moreover, the resulting ultra-high pulse dose rates of about $10^{10} \mathrm{~Gy} \mathrm{~s}^{-1}$, that exceed those of conventional accelerators by several orders of magnitude, demand for the investigation of potential consequences on the radiobiological effects of laser driven particle beams. The development of the new laser based technology towards clinical application has to follow the translational research chain from bench to bedside, i.e. starting with basic investigations followed by cell irradiation, animal experiments and pre-clinical trials.

The long-term goal of the German joint research project "onCOOPtics" is the research and development of laser based proton and ion accelerators for particle therapy of human cancer. In the framework of this project, the laser based technology was developed and established for cell irradiation experiments as the first translational step. Its stable and reliable application was proven in systematic radiobiological studies, firstly with laser accelerated electrons at the laser system JETI at FSU $[1,2,3]$ and secondly with laser accelerated protons at the laser system DRACO at HZDR [4,5].

As the next step in the translational chain, animal experiments have to be established, which means the translation of the laser based irradiation technology from 2D (cell monolayer) to 3D (tumour volume) dose delivery. The corresponding technological development was for the first 
time successfully realized for laser accelerated electrons at JETI. The laser based irradiation system described here includes all key components, e.g. laser electron accelerator, beam transport system including beam energy filtration, real-time beam monitoring and precise absolute dosimetry. Its stable and reliable operation was demonstrated by the irradiation of small human tumours on mice ear within ongoing radiobiological experiments.

\section{Methods}

\subsection{The Jena Titanium:Sapphire (JETI) laser accelerator}

The electron pulses were generated using the $30 \mathrm{TW}$ Ti:Sapphire laser system JETI that provides $28 \mathrm{fs}$ short pulses (700 mJ energy, $800 \mathrm{~nm}$ central wave length) at a pulse frequency of $1 \mathrm{~Hz}$. Focused into a hydrogen gas jet (Figure 1) the laser pulses produce plasma and accelerate electrons in the forward direction to energies of a few $10 \mathrm{MeV}$. In the following, the transport and properties of the generated electron beam had to be optimized in order to perform radiobiological experiments.

\subsection{Beam transport and irradiation field formation}

Following generation, the electron beam was filtered by a permanent magnet system that consist of two dipole magnets $(B=0.06 \mathrm{~T})$ of opposing polarity in order to remove the radiobiological more effective low-energy electrons $(\mathrm{E}<3 \mathrm{MeV})$. Subsequently, the electron beam enters a collimator system (c.f. Figure 1) consisting of a $118 \mathrm{~mm}$ aluminium block with an inner diameter of $10 \mathrm{~mm}$. At the beam entrance a scintillator screen was included that enables the online monitoring of the beam position by means of a CCD camera. The beam exited the collimator system

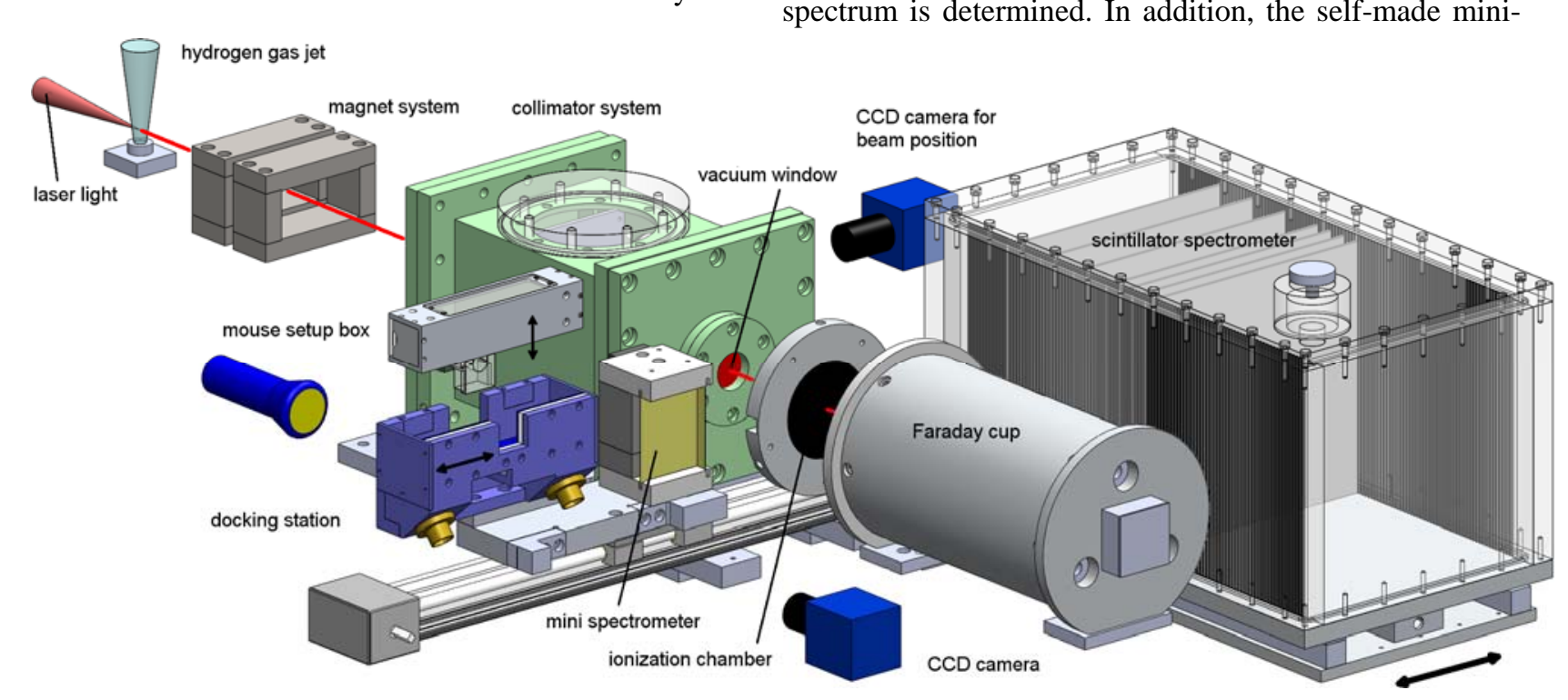

Figure 1 Schematic overview of the experimental setup for the irradiation of mice at the JETI laser system. through an $1 \mathrm{~mm}$ thick aluminium vacuum window and propagated in air by reason that the irradiation of living samples demand for atmospheric pressure.

For an optimum beam transport, i.e. a sufficient high intensity and adequate homogeneity over the irradiation the horizontal and vertical plane employing the hydrogen gas jet as beam origin and as rotation centre, respectively. During this process, the beam position relative to the central beam axis was monitored via the scintillator screen at the beam entrance.

\subsection{Real-time beam monitoring and abso- lute dosimetry at the irradiation site}

Based on the experiences of previous cell experiments at JETI $[1,2,3]$ a beam monitoring and dosimetry system consisting of a Faraday cup, a Bragg peak ionization chamber (PTW, Germany), Gafchromic ${ }^{\circledR}$ EBT-2 dosimetry films (ISP Corp., USA), a scintillator based spectrometer for high energy particles and a minispectrometer for low energy particles consisting of permanent magnets was established at the JETI system (c.f. Figure 1). At first, the Bragg peak chamber and the EBT2 films were used for beam optimization with regard to maximum beam intensity and to the homogeneous dose distribution over the irradiation field of about $5 \mathrm{~mm}$ in diameter, respectively. Later on, the Bragg peak chamber and the Faraday cup (200 mm steel, in house development and manufacturing) provide an online dose information, whereas EBT-2 films in front of the tumour were applied for retrospective precise dose determination and dose homogeneity control.

Electron spectra in real-time were obtained by means of the scintillator spectrometer (in-house production, liquid scintillator BC $517 \mathrm{H}$ ), where the radiation induced scintillation light was monitored with a CCD camera providing information about the depth dose distribution (up to spectrum is determined. In addition, the self-made minifield, the collimator system can be rotated and adjusted in $40 \mathrm{~cm}$ water equivalent depth). From those the electron 
spectrometer consisting of magnets and EBT-2 films provides retrospective energy information for electrons up to $20 \mathrm{MeV}$. For this, the laser electrons were deflected by the magnets, whereas the resulting spot positions, i.e. the deflection angles, on the EBT-2 films were used to ascertain their energy. The two spectrometers as well as the Faraday cup were movable to beam position along a linear axis, whereas the Bragg peak chamber has a fixed position and can be used in parallel to the Faraday cup and the scintillator spectrometer.

\subsection{Experimental setup for the in vivo irradiation of mice}

First animal experiments with laser accelerated electrons were performed on a mouse model that exhibits a small tumour on the right ear. For its precise and reproducible positioning at the irradiation site a system for mouse fixation, tumour positioning and position verification was implemented. At first, the anaesthetized mouse was placed in face-down position in the mouse setup box (Figure 2). The right ear was fixed on the sidewise attached PMMA block positioning the tumour over a milled circle of $5 \mathrm{~mm}$ in diameter that marks the target position. A (lead) aperture and an EBT-2 film holder above the tumour were also included in the setup box in order to reduce the out of field dose and to enable the retrospective precise dose determination, respectively.

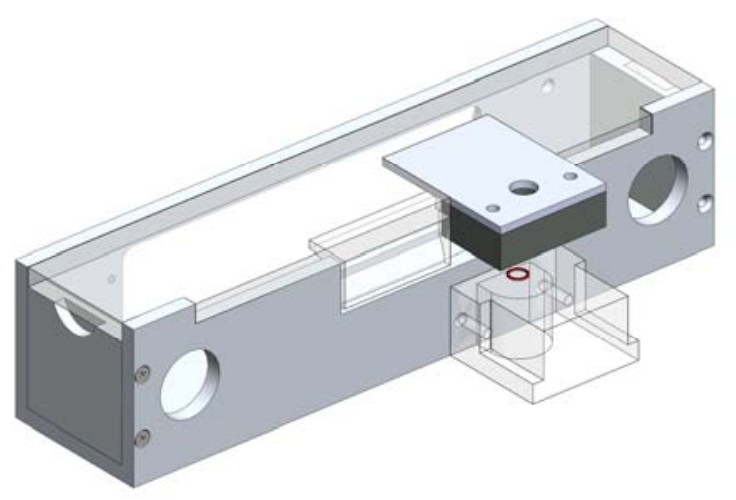

Figure 2 Mouse setup box for mouse fixation and tumour positioning; the milled ring that marks the target area of $5 \mathrm{~mm}$ in diameter is shown in red.

For irradiation, the setup box was inserted in the docking station facing the mouse ear downwards (blue box, Figure 1). Mounting the station on a linear axis allowed for a reproducible tumour positioning at the irradiation site. However, since the tilt of the setup box might cause positional changes of both mouse and tumour, online verification of the tumour position was necessarily included. For this, light from a source behind the docking station was shining through the transparent PMMA block and the thin mouse ear. As result, the tumour and the milled target area became observable with a CCD camera in front of the docking station and their relative positioning to each other can be controlled (Figure 3).
Besides the precise positioning of the small tumour in the beam, a further challenge for the experimentation with mice was the required temperature stability. Since anaesthetized mice are not able to regulate their body temperature the docking station and therewith the mouse setup box could be heated up keeping temperatures at $37^{\circ} \mathrm{C}$ during irradiation. Moreover, the ambient temperature during the whole mouse handling, i.e. transport, preparation, positioning and irradiation, was controlled and recorded by micro loggers SL52 (IMEC Messtechnik $\mathrm{GmbH}$, Germany) that were always carried close to the mouse.

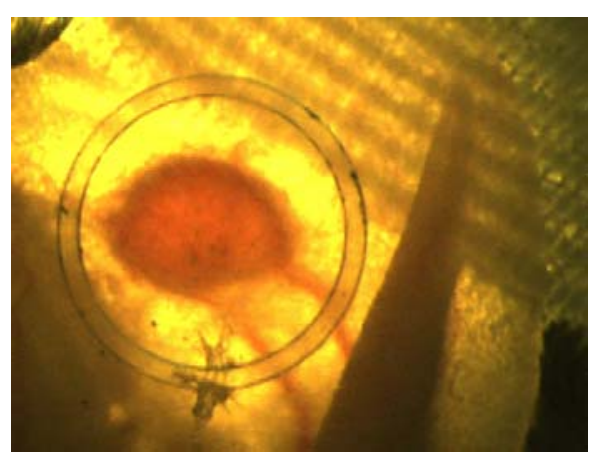

Figure 3 Relative positioning of the tumour and the milled target area.

\section{$3 \quad$ Results}

For the first time a laser based irradiation system was developed and established for animal experiments already comprising all the key components necessary for further steps in the translational chain. Magnetic beam filtering and a beam collimation system enabled an efficient beam transport that fulfils the requirements on beam intensity and dose homogeneity over the irradiation field. Realtime monitoring of essential beam parameters allow for interventions just in time and for the delivery of prescribed doses. Moreover, absolute dosimetry provides the precise dose administered to the tumour volume.

The presented laser based irradiation system was successfully applied for pre-clinical animal experiments, especially for the irradiation of small tumours on the ear of mice. The setup enables not only the precise and reproducible positioning of the tumour on beam axis, but also provides constant ambient conditions (temperature, sterility) to the mice.

All components combined, i.e. the advanced laser system JETI, the electron acceleration, the irradiation system and the setup for the mice were commissioned and successfully applied for ongoing systematic radiobiological studies over months. The whole system was found to meet all requirements for radiobiological experiments and to be stable, practicable and easy to handle, what results in reasonable times of about 15 min for the whole procedure of mouse preparation, transport and irradiation. 


\section{Conclusion}

The key components of the present laser based irradiation system, like laser accelerator, beam transport, beam monitoring and dosimetry and the reproducible positioning of the target volume at beam axis, are already relevant for further translational steps, including other (larger) animal models. In consequence, the first successful establishment of all necessary technical requirements for and the performance of systematic animal studies with laser accelerated electrons mark an important step towards the clinical application of laser accelerated particle beams.

\section{Acknowledgement}

The work was supported by the German Federal Ministry of Education and Research (BMBF), grant no. 03ZIK445. The authors are grateful to the JETI operator crew for their continuing interest and support of during the laser experiments.

\section{References}

[1] Beyreuther E, Enghardt W, Kaluza M et al. Establishment of technical prerequisites for cell irradiation experiments with laser-accelerated electrons. Med Phys 2010; 37(4): 1392-1400.

[2] Laschinsky L, Baumann M, Beyreuther E et al. Radiobiological effectiveness of laser accelerated electrons in comparison to electron beams from a conventional linear accelerator. J Radiat Res 2012; 53(3): 395-403.

[3] Richter C, Kaluza M, Karsch L et al. Dosimetry of laser-accelerated electron beams used for in vitro cell irradiation experiments. Radiat Meas 2011; 46(12): 2006-2009.

[4] Kraft SD, Richter C, Zeil K et al. Dose-dependent biological damage of tumour cells by laser-accelerated proton beams. New J Phys 2010; 12: 085003.

[5] Richter C, Karsch L, Dammene Y et al. A dosimetric system for quantitative cell irradiation experiments with laser-accelerated protons. Phys Med Biol 2011; 56(6): 1529-1543. 